\title{
Key Issues in Hymenoptera Venom Allergy: An Update
}

\author{
Alfaya Arias T', Soriano Gómis V², Soto Mera $\mathrm{T}^{3}$, Vega Castro $\mathrm{A}^{4}$, \\ Vega Gutiérrez JM ${ }^{5}$, Alonso Llamazares $A^{4}$, Antolín Amérigo $D^{6}$, \\ Carballada Gonzalez FJ', Dominguez Noche $C^{8}$, Gutierrez Fernandez $D^{9}$, \\ Marques Amat $\mathrm{L}^{10}$, Martinez Arcediano $\mathrm{A}^{11}$, Martinez San Ireneo $\mathrm{M}^{12}$, \\ Moreno Ancillo $A^{13}$, Puente Crespo $Y^{14}$, Ruiz Leon $B^{15}$, Sánchez Morillas $L^{16}$ \\ (Hymenoptera Allergy Committee of the SEAIC)
}

${ }^{1}$ Hospital General Universitario de Ciudad Real, Ciudad Real, Spain

${ }^{2}$ Hospital General Universitario de Alicante, Alicante, Spain

${ }^{3}$ Complejo Hospitalario de Pontevedra, Pontevedra, Spain

${ }^{4}$ Hospital Universitario, Gerencia de Atención Integrada de Guadalajara, Guadalajara, Spain

${ }^{5}$ Hospital Universitario Río Hortega, Valladolid, Spain

${ }^{6}$ Hospital Universitario Príncipe de Asturias, Alcalá de Henares, Spain

${ }^{7}$ Hospital Universitario Lucus Augusti (HULA), Lugo, Spain

${ }^{8}$ Hospital Virgen del Puerto, Plasencia, Spain

${ }^{9}$ Hospital Universitario Puerta del Mar, Cádiz, Spain

${ }^{10} \mathrm{Hospitales} \mathrm{Universitarios} \mathrm{Santa} \mathrm{María} \mathrm{y} \mathrm{Arnau} \mathrm{de} \mathrm{Vilanova,} \mathrm{Lleida,} \mathrm{Spain}$

${ }^{11}$ Hospital Universitario Araba (HUA), Vitoria-Gasteiz, Spain

${ }^{12}$ Hospital Virgen del Valle, Toledo, Spain

${ }^{13}$ Hospital Nuestra Señora del Prado, Talavera de la Reina, Spain

${ }^{14}$ Hospital Universitario Virgen Macarena, Sevilla, Spain

${ }^{15}$ Hospital Universitario Reina Sofía, Córdoba, Spain

${ }^{16}$ Hospital Universitario Clínico San Carlos, Madrid, Spain

J Investig Allergol Clin Immunol 2017; Vol. 27(1): 19-31

doi: 10.18176/jiaci.0123

\section{Abstract}

In this review, the Hymenoptera Allergy Committee of the SEAIC analyzes the most recent scientific literature addressing problems related to the diagnosis of hymenoptera allergy and to management of venom immunotherapy. Molecular diagnosis and molecular risk profiles are the key areas addressed.

The appearance of new species of hymenoptera that are potentially allergenic in Spain and the associated diagnostic and therapeutic problems are also described.

Finally, we analyze the issue of mast cell activation syndrome closely related to hymenoptera allergy, which has become a new diagnostic challenge for allergists given its high prevalence in patients with venom anaphylaxis.

Key words: Hymenoptera. Molecular diagnosis. Venom immunotherapy. Polistes dominula. Mastocytosis. 


\section{Resumen}

En esta revisión el Comité de Alergia a Himenópteros de la SEAIC ha analizado la literatura científica más reciente sobre los principales problemas diagnósticos de la alergia a himenópteros, así como sobre las dificultades que pueden surgir durante la inmunoterapia con venenos. Se revisan especialmente las novedades relacionadas con el diagnóstico molecular y los perfiles moleculares de riesgo. También se describe la alergia a himenópteros poco habituales y los problemas diagnósticos y terapéuticos que esta conlleva. Por último, se tratan los síndromes de activación mastocitaria clonal, íntimamente relacionados con la alergia a himenópteros, que se han convertido en un nuevo reto diagnóstico para el alergólogo.

Palabras clave: Himenópteros. Diagnóstico molecular. Inmunoterapia con venenos. Polistes dominula. Mastocitosis.

\section{Introduction}

Systemic reactions (SR) due to hymenoptera venom allergy affect between $2.3 \%$ and $2.8 \%$ of the rural population in Spain [1] and may lead to potentially life-threatening anaphylaxis.

A quality of life questionnaire specific to this disorder that was recently validated in Spanish [2,3] revealed that the quality of life of venom-allergic patients is poor owing to the anxiety caused by the risk of accidental exposure to insect stings.

The diagnosis of venom allergy is based on the clinical history and on demonstration of specific IgE against the venom responsible by means of intradermal skin and/or in vitro tests $[4,5]$. However, 2 problems frequently arise in diagnosis: first, patients may be sensitized to multiple venoms; and second, some cases of SR due to insect stings may be accompanied by negative skin and specific $\operatorname{IgE}$ blood test results. Recently developed molecular diagnostic tests and the basophil activation test (BAT) have proven useful in the resolution of some cases.

Venom immunotherapy (VIT) has been shown to be more than $80 \%$ effective in patients treated and is considered the ideal model for the study of the mechanisms of induction of immune tolerance produced by specific immunotherapy with allergens [4]. Furthermore, it has been shown to improve the quality of life of venom-allergic patients when compared with patients who do not receive immunotherapy but carry adrenaline [6].

The management of adverse reactions caused by VIT, the monitoring of the efficacy of VIT, and the procedure to be followed in cases of therapeutic failure are common problems in everyday clinical practice that have been widely debated in recent publications.

Closely related to hymenoptera allergy, mast cell activation syndrome has become a new diagnostic challenge for allergists given its high prevalence in patients with venom anaphylaxis.

Finally, it is also important to remember the appearance of new, potentially allergenic species of hymenoptera in Spain, and the diagnostic and therapeutic difficulties involved in managing stings by these species.

In this review, the Hymenoptera Allergy Committee of the SEAIC analyzes the most recent scientific literature dealing with the problems described above, together with other controversial aspects of the management of hymenoptera venom allergy, with the aim of updating what is known about this disorder. Furthermore, special attention has been paid to problems that specifically affect Spain, such as the abundance of species of the Polistes genus, which are relatively scarce in the north of Europe.

\section{Difficulties in the Diagnosis of Insect Venom Allergy}

\section{Latest Advances in the Molecular Diagnosis of Insect Venom Allergy}

Molecular techniques play a key role in the diagnosis of hymenoptera allergy by determining the insect responsible and, thus, the composition of immunotherapy. In recent years, significant advances have been made in the identification of new allergens and the development of techniques for recombinant production of allergens.

The best-characterized venom is that of the bee, Apis mellifera. Twelve allergens have been identified to date [7] (Table 1). The best known are phospholipase A2 (Api m 1), which is the most potent allergen in venom $[8,9]$. Both Api 1 and hyaluronidase (Apim 2) are considered major allergens [8]. Together with melittin (Api m 4), they make up most of the dry weight of the venom. The peptide melittin (Api m 4) is considered to be an allergen with low prevalence $[8,10]$. However, its relevance has recently been demonstrated, and it has been proposed as a biomarker of poor tolerance in patients at the initial stages of immunotherapy [11]. Melittin also makes it possible to distinguish between 2 forms of allergy to bee venom [12]. Other relatively scarce proteins that have been reported to be potentially allergenic are acid phosphatase (Api m 3) [8,13], dipeptidylpeptidase IV (Api m 5) [14], and icarapin (Api m 10), a complex protein with various isoforms [15]. Icarapin is a real allergen of bee venom of great diagnostic interest that could be under-represented in some therapeutic extracts $[8,16]$.

With regard to vespids, the 2 predominantly allergenic species in Spain are Polistes dominula and Vespula germanica. For diagnosis, it is important to use allergens from both species, especially Polistes dominula, as their sensitivity and specificity are greater than those of allergens from American Polistes species [17].

Diagnostic tests yield double-positive results to both vespids in approximately $50 \%$ of cases; these results are more common in the in vitro determination of $\operatorname{IgE}$ [18]. The clinical history may help to determine the insect responsible, although 
Table 1. Allergens of Venom From Apis mellifera, Polistes dominula, and Vespula vulgaris

\begin{tabular}{|c|c|c|c|c|c|c|}
\hline Allergens & Biological Function & $\begin{array}{l}\text { Molecular } \\
\text { Weight, }\end{array}$ & $\begin{array}{l}\text { Dry Fraction } \\
\text { of Venom, \% }\end{array}$ & $\begin{array}{l}\text { Positive } \\
\text { sIgE, } \%\end{array}$ & Glycosylation & $\begin{array}{l}\text { Eucaryotic } \\
\text { Expression }\end{array}$ \\
\hline Api m 1 & Phospholipase A2 & 16 & $7-15$ & 95 & Yes & Yes \\
\hline Api m 2 & Hyaluronidase & 43 & $1-3$ & 50 & Yes & Yes \\
\hline Api m 3 & Acidic Phosphatase & 45 & 1 & 37 & Yes & Yes \\
\hline Api m 4 & Melittin & 2.8 & $35-50$ & $29 / 56$ & No & No \\
\hline Api m 5 & Dipeptidylpeptidase IV & 102 & 1 & 60 & Yes & Yes \\
\hline Api m 6 & Protease Inhibitor & 8 & $1-2$ & 42 & No & Yes \\
\hline Api $m 7$ & Protease & 39 & $<1$ & 80 & Yes & Yes \\
\hline Api $m 8$ & Carboxylesterase & 70 & $<1$ & $?$ & Yes & Yes \\
\hline Api m 9 & Carboxypeptidase & 60 & $?$ & $?$ & Yes & Yes \\
\hline Api m 10 & Icarapin & $50-55$ & $?$ & 50 & Yes & Yes \\
\hline Api m 11 & Major royal jelly protein & $50-60$ & $?$ & $15 / 34$ & Yes & Yes \\
\hline Api m 12 & Vitellogenin & 200 & $?$ & 40 & Yes & Yes \\
\hline Pol d 1 & Phospholipase A1 & 34 & $?$ & 87 & No & No \\
\hline Pol d 2 & Hyaluronidase & 44 & $?$ & $?$ & Yes & No \\
\hline Pol d 4 & Protease & 33 & $?$ & $?$ & Yes & No \\
\hline Pol d 5 & Antigen 5 & 23 & $?$ & 66 & No & Yes \\
\hline Ves v 1 & Phospholipase A1 & 35 & $6-14$ & 79 & No & Yes \\
\hline Ves v 2 & Hyaluronidase & 45 & $1-3$ & 32 & Yes & Yes \\
\hline Ves v 3 & Dipeptidylpeptidase IV & 100 & 1 & $?$ & Yes & Yes \\
\hline Ves v 4 & Protease & 42 & $?$ & $?$ & Yes & No \\
\hline Ves v 5 & Antigen 5 & 25 & $5-10$ & 87 & No & Yes \\
\hline Ves v 6 & Vitellogenin & 200 & $?$ & $?$ & Yes & Yes \\
\hline
\end{tabular}

very often it is necessary to turn to molecular diagnosis. The allergens most widely used for diagnosis are the phospholipases (Pold 1 and Ves v 1) and antigen 5 (Pol d 5 and Vesv 5) [19]. The hyaluronidases (Pol d 2 and Ves v 2) are minor allergens and, given their similarity with bee hyaluronidase (Api m 2) [20], especially through carbohydrate determinants [21], may contribute to the double positivity observed between bees and wasps. Other potentially relevant allergens are protease in Polistes (Pol d 4) and dipeptidylpeptidase IV (Ves v 3 ) and vitellogenin (Ves v6) in Vespula, which exhibit partial identity with the bee allergens dipeptidylpeptidase IV (Api m 5) and vitellogenin (Api m 12), respectively [14,22].

Apim 1, Apim 10, Vesv 1, Ves v 5, and Pol d 5 are available in recombinant form for routine molecular diagnosis. In the case of wasps, it is necessary to use the allergens of the 2 insects to guarantee a good differential diagnosis [17]. The sensitivity of Api 1 for the diagnosis with bee venom is highly variable and depends on the selection criteria for the population, the technique applied, and the type of molecule used [23-26]. In a recent study, the incorporation of new specific recombinant allergens from bee venom (Api m 2, Api m3, Api m 4, Api m 5, and Api m 10) increased sensitivity to $95 \%$ and demonstrated that these allergens (with the exception of Api m 4) may also sensitize more than $50 \%$ of the population [8].

\section{Systemic Reactions Due To Hymenoptera Stings With Negative Skin and In Vitro IgE Test Results}

The diagnosis of hymenoptera venom allergy is based on the combination of a clinical history compatible with SR following a sting and the demonstration of an IgE-mediated mechanism by means of positive intradermal skin tests and/or in vitro identification of specific IgE against the venom [4]. However, $4 \%$ to $6 \%$ of patients experiencing SR following an insect sting have negative skin test results and no detectable specific IgE in serum [27]. These patients represent a diagnostic challenge, it is essential to know which venom the patient is sensitized to before immunotherapy with venoms can be prescribed.

The main causes for negative test results in patients with SR due to stings are as follows:

- The tests are performed in the refractory period following the sting reaction.

- The reaction is not recent and, as such, spontaneous desensitization has occurred with the passing of time.

- The tests are performed with the inappropriate venoms.

- The patient experienced a vasovagal or toxic reaction with no underlying allergic mechanism.

- The allergen to which the patient is sensitized is not adequately represented in the extract analyzed. 
- The presence of systemic mastocytosis, as negative results occur in up to $15 \%$ of these patients [28].

Consequently, the initial approach for such cases is to repeat the skin and blood tests once the refractory period of 2 weeks has passed. It is advisable to perform the tests 1-2 months after the reaction [5] and to extend the tests to include other available venoms.

The use of recombinant venom allergens for diagnosis allows the identification of allergens that are under-represented in the commercial venoms, as is the case with Api m 3 and Api 10 in bee venom. Indeed, it has been calculated that up to $5 \%$ of patients sensitized to bee venom could have a negative result in normal diagnostic tests [8,16,29].

Some patients have negative specific $\operatorname{IgE}$ to wasps in commercial serologic tests but have positive results to Ves v 1 , Ves v 5, Pold 1, or Pol d 5 [17]. In a sample of 308 patients with SR due to Vespula stings, Vos et al [30] found that only $83.4 \%$ of the patients had positive specific IgE, as measured by ImmunoCAP with Vespula venom. However, when molecular allergens such as Ves v 5 were used, as many as $96 \%$ of patients were sensitized, with the levels of specific IgE to Ves v 5 being much higher than the levels of specific IgE to Vespula. This suggests that the level of Ves $v 5$ in the extract of the whole venom was under-represented.

Therefore, molecular diagnosis may be useful for patients with undetectable levels of specific IgE both in skin tests and serum, as it enables sensitization to allergens under-represented in the whole extract to be identified.

A cutoff point of $0.10 \mathrm{kU}_{\mathrm{A}} / \mathrm{L}$ in ImmunoCAP with venom allergens was recently shown to increase sensitivity in patients with negative results [19].

Another increasingly useful test in the diagnosis of hymenoptera venom allergy is the BAT, especially with the CD63 activation marker [4]. This test has a diagnostic sensitivity of between $85 \%$ and $100 \%$, with a specificity of $83 \%$ to $100 \%$, depending on the study [27]. It has been reported that BAT is capable of diagnosing up to two-thirds of patients with SR due to stings and negative results with conventional tests $[27,31]$.

The problem of negative test results in patients with mastocytosis will be analyzed in a separate section of this review.

\section{Multiple Sensitizations to Hymenoptera Venoms}

Some patients exhibit multiple sensitizations, either to bees and wasps (in up to $59 \%$ of cases [9]) or between different wasps such as Vespula and Polistes (in up to 50\% of cases [18]). In such cases, allergists need to know whether they are faced with real double sensitization or cross-reactivity, where the primary source of sensitization must be determined. This is particularly important, as real double sensitization requires specific immunotherapy with both venoms.

It is possible that the $\mathrm{IgE}$ recognizes the glycosylated portion of the venom proteins, ie, the cross-reactive carbohydrate determinants (CCD) [32-37], thus suggesting cross-reactivity of doubtful clinical relevance. Identification of specific IgE against MUFX enables positive results to the CCD to be detected (by means of ImmunoCAP in 59\% of cases and the ADVIA platform in $32 \%$ of cases).
There may also be cross-reactivity between homologous allergens from different species [14,38] if they partially share their identity, as is the case with hyaluronidase (Api $\mathrm{m} \mathrm{2/}$ Ves v 2), dipeptidylpeptidase IV (Api m 5/Ves v 3), and vitellogenin (Api m 12/Ves v 6). The relevance of these crossreactions remains unknown, and further studies are needed to clarify the issue.

The techniques used to distinguish between double sensitization and cross-reactivity are as follows:

1. Radioallergosorbent test/CAP inhibition. This is a useful technique [39] that allows real multiple sensitizations to be identified; however, it is not always available in all centers and requires large amounts of serum and, generally, specific IgE levels $>5 \mathrm{kU}_{\mathrm{A}} / \mathrm{L}$. Furthermore, in the case of wasps, it has been reported that this technique was not able to determine the main sensitizing trigger in $31 \%$ of cases [40].

2. Molecular diagnosis or determination of specific $\operatorname{IgE}$ against the isolated allergenic components of the venoms (native or recombinant) is a useful tool that is effective in differentiating between double sensitization and crossreactivity $[17,41,42]$.

- Bee vs Vespula. It has been demonstrated that the use of rApi m 1 on its own is insufficient to distinguish between bee and wasp venom [43]. The use of other bee allergens, such as rApi 2 and nApi m 4, or more recently, rApim 3 and rApim 10, improves the detection of sensitization to bee venom [41-44], although the use of rApim 1 and rVesv 5 is insufficient to clearly identify double-positive results [23]. With regard to Vespula venom in these cases, the combination of rVes $\mathrm{v} 1$ and rVes v 5 improves the diagnosis [44].

- Vespula vs Polistes. Diagnosis has improved using venom from Polistes dominula and the native antigens represented in the main allergens [17]. In an extensive study carried out by the Committee on Hymenoptera Allergy using the ADVIA Centaur platform, the use of the antigen 5 vespid recombinants (rVes v5 and $\mathrm{rPol} \mathrm{d} 5$ ) only enabled specific IgE to be identified in $52 \%$ of patients, while the use of native antigens and Polistes dominula (nVes v 5 and $\mathrm{nPol}$ d 5) increased sensitivity to $80 \%$. Moreover, the addition of other native antigens (Pold 1 and Ves v 1) made it possible to detect IgE in $100 \%$ of patients [17]. Simultaneous use of the phospholipases (nVes v 1 and nPold 1) and antigen 5 (nVes v 5 and nPol d 5) from the venoms of Vespula and Polistes enabled identification of the sensitizing vespid in $69 \%$ of cases [17]. Therefore, the main allergens of Polistes dominula and Vespula vulgaris (phospholipases and antigen 5) are required to discriminate between sensitizing species.

3. The BAT, which is more expensive and more timeconsuming than other techniques, is still not fully standardized and is also activated with CCDs, although these determinants influence this technique less than they do determination of IgE to whole venom [33]. While the BAT serves to detect the existence of double sensitization better than IgE to whole venom $[27,45]$, it is more useful in the diagnosis of SRs with negative skin and specific IgE tests. 
Once double sensitization has been confirmed, treatment should be initiated with the venoms separately. Administration of the vaccines of the 2 species of vespids over alternate months has been used successfully, with this format achieving the same efficacy as that offered by monthly conventional treatment [46].

\section{Difficulties in the Management of Immunotherapy With Hymenoptera Venom}

\section{Systemic Reactions Caused by Venom Immunotherapy}

SRs involving respiratory and/or cardiovascular symptoms can occur during VIT and occasionally require emergency interventions. However, VIT-related severe adverse effects are very infrequent, as reflected by the fact that only one-third of SRs usually require treatment [47]. Conflicting results mean that risk factors for such severe systemic adverse effects during the buildup phase of VIT continue to be a matter of debate (Table 2) [48].

Table 2. List of Potential Risk Factors for Adverse Effects During VIT (Modified From $[48,105]]^{\mathrm{a}}$
- Honey bee $>$ vespid venom
- Buildup phase>maintenance phase
- Dose increase schedule (ultrarush)
- Treatment with ß-blockers/angiotensin-converting enzyme inhibitors
- Clonal mast cell activation syndrome
- Elevated baseline serum tryptase (surrogate marker for anaphylaxis)
- Low levels of preexisting specific IgG antibodies

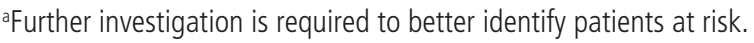

The observational prospective multicenter study (680 patients) performed by the Insect Venom Allergy Interest Group of the EAACI [49] aimed to characterize independent predictors of a higher complication rate during VIT. The study identified several risk factors: honeybee VIT; the type of dose increase (ultrarush) during therapy; accompanying antihypertensive therapy; and baseline serum tryptase (BST) concentration for vespid-allergic patients; and the presence of venom-specific IgE in serum. Another important aspect was the possible association between emergency interventions and serum specific IgE levels. Such is the case of challenge tests with live insects in patients who do not receive VIT, in whom a correlation with the frequency of severe anaphylactic reactions was reported [49].

In addition to $\mathrm{IgE}$, antigen-specific $\mathrm{IgG}$ has been associated with the frequency of severe adverse effects during VIT. It is well known that exposure to the elicitor causes a temporary rise in antigen-specific IgG, which lasts for several weeks. Likewise, there is evidence that specific IgG may block IgE- mediated anaphylaxis. During buildup phases, when low antigen doses are used, pre-existing high IgG levels were found to prevent anaphylactic responses [50].

Bee venom allergy is an independent predictor for higher risk during VIT, and vespid venom allergy was shown to be an independent predictor for a higher risk of a severe SR after a field sting. Remarkably, the severity of the preceding field sting reaction did not correlate with the extent of adverse effects during VIT [51].

Rueff et al [49] found a cluster of adverse effects in the $1-\mu \mathrm{g}$ to $30-\mu \mathrm{g}$ dose range during the buildup phase of bee VIT. In another study (840 patients), factors such as female sex, rapid dose-increase regimens, treatment with bee venom extract, and pre-existing allergic rhinitis seemed to increase the risk of adverse effects of VIT [47].

The most abundant peptide in bee venom is melittin (Api m 4), which has a very low molecular weight and is the major toxic component. It is considered a minor allergen, with a prevalence of sensitization ranging from $22.9 \%$ to $29 \%[8,11]$. However, it was recently described as a major allergen in other populations [12]. Sensitization to Api m 4 has been proposed as a candidate biomarker of poor tolerance in patients at the initial stages of bee venom immunotherapy, even from very low specific IgE values, and was shown to discriminate between 2 different ways of being allergic to bee venom $[8,11]$.

Much debate surrounds the suitability and efficacy of pretreatment in terms of preventing SR to VIT. Brockow et al [52] showed that antihistamine pretreatment was associated with a much lower rate of discontinuation of VIT as a result of SR, a finding that contradicts those of previous studies [53]. Moreover, pretreatment with antihistamines can also increase the efficacy of immunotherapy, probably by inhibition of type 2 helper T-cell cytokine production [48].

Isolated cases of SR during immunotherapy in which premedication with omalizumab has enabled treatment to be completed have been reported. However, the most appropriate doses of omalizumab and the intervals between administrations have yet to be established [54-57].

Both purified and nonpurified extracts are available for induction of tolerance in venom-allergic patients. Purified extracts appear to be better tolerated than nonpurified products and may improve diagnostic procedures in patients with suspected insect venom allergies [58,59].

Purified aqueous venom extracts have no low-molecularweight components, namely, vasoactive amines such as dopamine, histamine, and serotonin, which may be included in the native venom extract. Purification of these extracts warrants separation of the protein fractions by means of their molecular weight. Moreover, gel filtration procedures reduce the presence of small peptides such as apamin, kinins, and mast cell degranulating peptides.

\section{Lack of Efficacy of Venom Immunotherapy}

The lack of efficacy, understood as the onset of an SR following a sting while the patient is in the maintenance phase or after completing treatment, is calculated to affect between $10 \%$ and $15 \%$ of patients treated [60] and has been associated with the following risk factors: 


\section{Underlying medical conditions}

After completing VIT, elderly patients are at greater risk of severe SR, both in the short and the long term. This is due, at least in part, to the coexistence of cardiovascular and respiratory disorders that increase the severity of anaphylaxis.

The influence of angiotensin-converting enzyme (ACE) inhibitors and $\beta$-blockers on the efficacy and safety of VIT continues to be controversial, and studies have yielded contradictory results [49,61-63]. Prospective studies including a representative proportion of individuals receiving these treatments are needed before definitive conclusions can be drawn.

\section{Allergy to bee venom}

With the standard dose, patients allergic to bee venom are at greater risk of SR when stung again during VIT (11\%) than those allergic to wasp venom $(4 \%)[61,63]$. Increasing the maintenance dose in these patients has been shown to provide complete protection during treatment in most cases [64].

Allergy to bee venom has also been associated with a greater risk of relapse after completion of immunotherapy [65]. There are several reasons for this difference. When wasps sting, they inject 3-5 $\mu \mathrm{g}$ of venom as compared with the $50 \mu \mathrm{g}$ injected by bees. Thus, with the same maintenance dose, patients with allergy to bee venom receive a proportionally much smaller amount [66]. Furthermore, it was recently demonstrated that in some commercial extracts used in VIT, allergens such as Api m 3, Api m 4, and Api m 10,are either not present or under-represented, thus explaining the incomplete protection observed, especially for the $5 \%$ of patients who are sensitized exclusively to these components [8].

\section{Severe reaction prior to VIT}

Patients with a severe reaction to a sting prior to VIT are at greatest risk of SR with subsequent stings, both while receiving and after completing VIT. Combining the data from different studies, severe relapses are observed in $4 \%$ of patients with mild SRs in contrast to $14 \%$ of those with severe SRs before treatment [67].

\section{Clonal mast cell activation syndrome}

Clonal mast cell activation syndrome is considered a risk factor both for experiencing SR in VIT and for severe and fatal reactions with a subsequent sting after interrupting treatment $[49,68,69]$. However, in the absence of mastocytosis, the association between BST and lack of efficacy of VIT is controversial. In a recent European multicenter prospective study, an increase in BST $(>11.4 \mu \mathrm{g} / \mathrm{L})$ by itself did not prove to be an independent risk factor either for serious adverse effects of VIT or for the lack of efficacy of VIT, although it was associated with a greater risk of SR in immunotherapy with Vespula venom [61].

Patients with clonal mast cell activation syndrome can be successfully treated with VIT, although experts recommend that they should receive lifetime VIT, as they are at greater risk of recurrence once treatment is discontinued $[69,70]$.

\section{Systemic reaction during VIT}

Experiencing an SR with a sting or as an adverse reaction during the administration of VIT indicates insufficient protection. In such cases, there is also a significantly greater likelihood of relapse $(13 \%$ vs $6 \%)$ once treatment is finished $[61,63]$. Most patients would be protected by increasing the maintenance dose [64].

\section{Frequency of exposure}

Once treatment is finished, repeated stings are associated with a greater risk of SR $[60,65]$. Therefore, in patients with frequent occupational or vocational exposure, it is necessary to weigh up the benefit of continuing treatment for a longer period.

\section{Time interval after interrupting VIT}

Although most patients remain sufficiently protected after interrupting VIT, there does seem to be a loss of efficacy over time, most frequently leading to mild SR. The rate of complete protection during the first 7 years after completing VIT is $80 \%-91 \%$ and is slightly higher when efficacy during the first 3 years after completing treatment is assessed [67].

After completion of VIT, the increase in the risk of SR due to stings is inversely proportional to the duration of VIT [60]

\section{Duration of VIT}

There is no evidence to indicate which patients are at risk of relapse after completing VIT. Most studies agree that 5 years is sufficient for most of those affected. However, bearing in mind that longer treatment periods are associated with a lower risk of relapse [60], in patients with associated risk factors (Table 3), thought must be given to prolonging treatment or even maintaining it indefinitely. Three years could be sufficient for mild reactions, when skin and specific $\operatorname{IgE}$ tests become negative, although such circumstances are rare [71-73].

Table 3. Measures to Increase the Efficacy of VIT

Duration of VIT

- Five years is acceptable for most patients with mild or moderate SRs.

- Consider prolonging VIT, even lifetime treatment, in patients with the following:

- Risk of serious SR: previous severe reaction, older age, cardiovascular disease, concomitant treatment with angiotensin-converting enzyme inhibitors or $\beta$-blockers, mastocytosis, and elevated baseline serum tryptase

- SR during VIT

- Exposure to repeated stings

- Negative effects on quality of life (such as anxiety, limitation of activities due to fear of unexpected stings)

Consider increasing the dose of VIT

- In bee venom allergy

- Patients with SR during VIT or field stings

Abbreviations: SR, systemic reaction; VIT, venom immunotherapy. 
A summary of the suggested interventions to improve the efficacy of VIT is given in Table 3.

\section{Monitoring Immunotherapy With Hymenoptera Venom}

VIT is effective in more than $80 \%$ of patients treated, although no in vitro techniques are able to predict the effectiveness of treatment in a specific patient [4].

Classically, monitoring of the efficacy of immunotherapy has been based on serial control with specific $\operatorname{IgE}$ and skin tests, although the test that has proven to be most effective is the sting challenge test (SCT).

Wheal size and specific IgE values are normally reduced 1 year after treatment; consequently, this approach is not useful for early monitoring [74]. Furthermore, there is not always a correlation between reduction in the results of these tests and clinical efficacy of treatment [75].

Monitoring IgG4 levels has also been used as a follow-up tool, as these levels increase during treatment. However, no correlation has been demonstrated with clinical efficacy, and the technique has therefore fallen into disuse [74,76].

The possibility of monitoring levels of $\operatorname{IgE}$ and specific IgG4 against the recombinant allergens of the venoms appears interesting, since component-based diagnosis has demonstrated greater sensitivity and specificity than IgE against whole extracts. However, at present, few data are available, and it has not been demonstrated that the reduction in $\operatorname{IgE}$ to components during VIT is correlated with clinical efficacy [19,77].

Several studies assess the usefulness of the BAT in the monitoring of VIT. These have shown significant reductions in the expression of the CD63 marker after only 6 months of treatment, that is, at an earlier stage than IgE and skin tests. In one study, the reduction in BAT was correlated with tolerance to stings after VIT was completed [27,78]. However, currently, BAT is not sufficiently standardized, and more studies are needed to establish its real usefulness in the monitoring of VIT.

Reduced expression of $\mathrm{CD}^{+} \mathrm{T}$-cell activation markers (CD30, CD145, and CD152) was recently identified after 4 and 6 months of VIT [79,80], although, again, it has not been possible to establish a correlation between these changes and the clinical effectiveness of treatment. Interesting as this approach may be, more studies are needed, and this technique is currently only available for research purposes.

The SCT is the test that has shown the highest correlation with the clinical efficacy of VIT [81], although it is cumbersome and not without risk for patients. Unlike controlled challenge tests with foods or drugs, the exact dose of venom administered is unknown, and administration with increasing doses is not possible. While there are reports of the injection of venom using a microsyringe to allow known and increasing doses of venom to be administered, this technique is not available for routine use [82]. Although false negatives can subsequently react with spontaneous stings, the SCT is generally considered to have a high predictive value for future stings. Furthermore, various studies have shown that the SCT improves patient quality of life, as it reduces the level of uncertainty associated with the risk of spontaneous stings [83-85].
Annual office follow-up is advisable to assess possible changes in medication that could affect the condition (eg, antihypertensive drugs) and clinical response to spontaneous stings. No consensus has been reached on the usefulness of repeating determination of specific IgE or skin tests annually or every 2 years [72].

From all of the above, it can be concluded that we still do not have an in vitro technique that would allow the effectiveness of VIT to be predicted. Therefore, allergists should use the SCT in those cases where it is necessary to prove clinical efficacy.

\section{Management of Anaphylaxis Due to Rare Hymenoptera (Bombus, Vespa)}

\section{Bombus}

Allergic reactions due to bumble bee stings (species of the Bombus genus) are not common in the general population, although their frequency has been increasing since the common bumble bee (Bombus terrestris, BT) has been used for the pollination of greenhouse plants, thus generating an occupational allergic disease for people working in such settings [86].

The allergens described in BT venom are Bom $t 1$ (phospholipase A2) and Bom t 4 (protease). In other species, the allergens are Bom p 2 (hyaluronidase) and Bom p3 (acid phosphatase) for Bombus pennsylvanicus and Bom hy12 (vitellogenin) for Bombus hypocrite [87]. The homology of amino acid sequences between the venoms of the different species of Bombus is high ( $>80 \%)$ and is logically lower than that of the amino acid sequences for Apis mellifera. Hoffman et al [86] found a homology of 53\% between the phospholipase A2 of both venoms, and recent studies have shown that $72 \%$ of the components detected in BT venom have a homolog in Apis mellifera [88].

Traditionally, immunotherapy with Apis mellifera venom has been recommended for BT-allergic patients based on the existing cross-reactivity and the lack of a specific BT extract [89]. The lack of identity described above may explain the failure of immunotherapy with Apis mellifera venom in some BT-allergic patients [90]. The availability of BT venom extract for diagnosis and immunotherapy solved this problem, although it seems that it will soon be removed from the market.

We can distinguish 2 types of BT-allergic patients [91,92]:

- Type I: patients with no occupational exposure and with primary sensitization to Apis mellifera venom. Such patients respond favorably to immunotherapy with Apis mellifera venom [93].

- Type II: patients with occupational exposure and sensitization specifically to BT venom. Since such patients have low cross-reactivity with Apis mellifera venom, immunotherapy with BT venom is recommended [93].

As a result, the availability of BT venom extracts for diagnostic tests and immunotherapy is essential for the management and treatment of these patients.

\section{Vespa}

Within this genus, the most representative species is Vespa crabro (VC), which is widely distributed across Asia, 


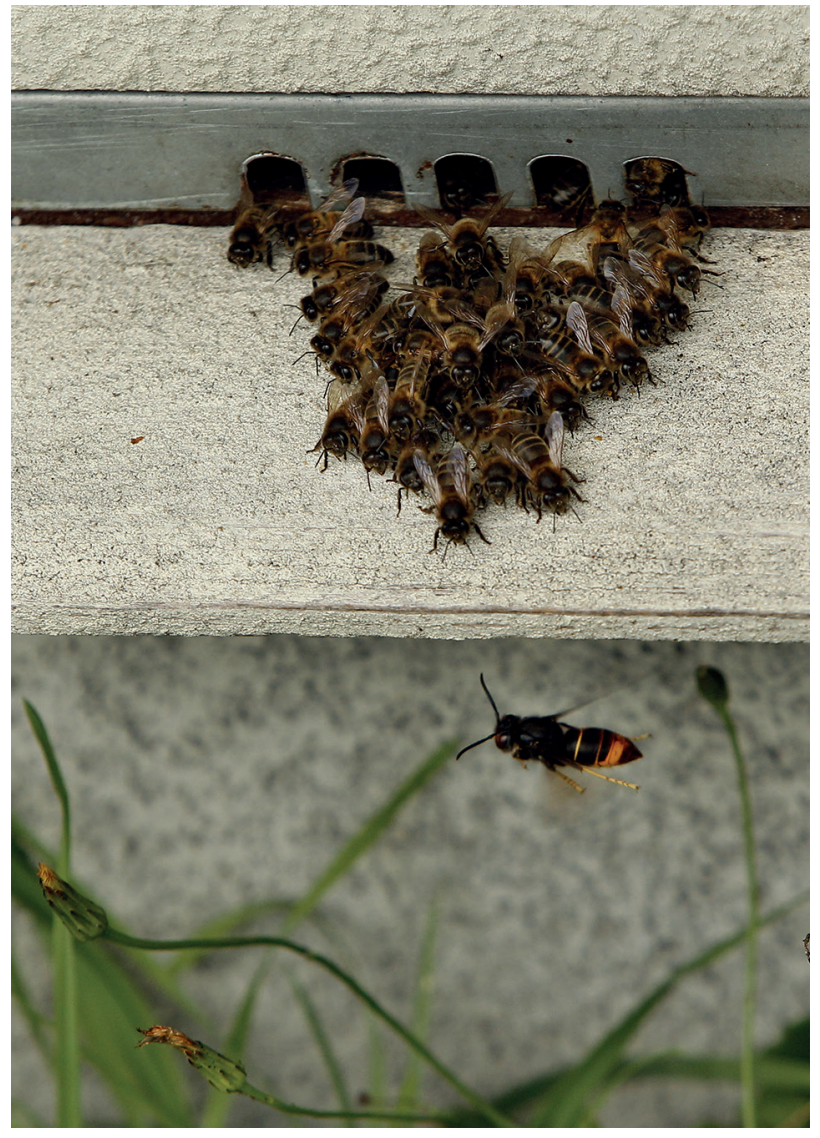

Figure. Position adopted by bees in the presence of Vespa velutina apparently defending and protecting their hive. Photo provided by José Alvez (Lugo).

Europe, and the USA. Invasive species from Asia that are also gaining in importance include Vespa orientalis (VO) and Vespa velutina, the Asian predatory wasp (Figure).

$\mathrm{VC}$ is responsible for particularly serious allergic reactions [94]. Isolated cases of anaphylaxis due to Vespa velutina $[95]$ and $\mathrm{VO}[96]$ have also been reported. The allergic reactions usually occur following previous stings from other vespids, especially from Vespula species.

The allergens described in VC venom are Vesp c 1 (phospholipase A1), Vesp c 2 (hyaluronidase), and Vesp c 5 (antigen 5) [87]. Marked cross-reactivity caused by antigen 5 is found between the venoms of Vespula, Dolichovespula, and Vespa $[95,97]$. However, hyaluronidase is probably the main antigen in $\mathrm{VO}$ venom, and there are cases of patients monosensitized to VC or VO venom [98].

For the diagnosis of anaphylaxis due to Vespa species, the only commercially available technique is determination of IgE against VC venom. Given the high cross-reactivity between the species, it is useful to perform intradermal tests and determination of specific IgE to whole extract and components (Ves v 1, Pold 1, Ves v 5, and Pol d 5) of the venom of other vespids.

Venom from species of the Vespa genus is not available for immunotherapy, and although cross-reactivity with other vespids is not complete, it is necessary to use those treatments that are available to protect our patients. Thus, when such a sensitization is found, immunotherapy with Vespula venom only should be sufficient $[93,98]$.

\section{Management of Indolent Systemic Mastocytosis and Anaphylaxis Due to Hymenoptera Venom}

The relationship between mastocytosis and anaphylaxis due to hymenoptera venom allergy has been clearly established.

Hymenoptera stings are the main triggers of anaphylaxis (excluding idiopathic cases) in patients with indolent systemic mastocytosis (ISM) [99,100], with a prevalence of 5\%-19\% $[99,101]$. These figures increase in specific forms of the disorder, such as ISM without skin lesions. The frequency of anaphylaxis following hymenoptera stings is $66 \%$, with an IgE-mediated mechanism being demonstrated in $87 \%$ of such cases [102].

Similarly, the prevalence of mastocytosis in patients allergic to hymenoptera venom is as high as $7.9 \%$, a figure much higher than that of the general population (3-13 cases per 100000 inhabitants).

ISM without skin lesions associated with anaphylaxis due to hymenoptera stings exhibits demographic, clinical, and laboratory characteristics that differ from those of other types of mastocytosis [103]. It predominantly affects men aged $30-40$ years. The main symptoms are cardiovascular, namely, dizziness, presyncope, tachycardia, hypotension, and shock. Up to half of all patients lose consciousness. The appearance of skin lesions is infrequent (in 78\% the skin is not affected), and symptoms caused by release of mastocyte mediators outside anaphylactic episodes caused by stings are infrequent. BST levels are normally lower than in other types of mastocytosis $(<20 \mu \mathrm{g} / \mathrm{L}$ in $50 \%$ of patients), and mutation of the kit receptor is restricted to mastocytes, with other hematopoietic cell lines being unaffected.

BST levels must be determined in all patients with anaphylaxis following a hymenoptera sting, especially in severe cases of anaphylaxis with hypotension and no skin lesions. The Spanish Mastocytosis Network (Red Española de Mastocitosis, REMA) has developed an algorithm based on clinical signs and symptoms and BST levels that is capable of predicting the existence of clonality [104] with a sensitivity of $92 \%$ and a specificity of $81 \%$ (Table 4 ). A score $\geq 2$ suggests that ISM should be ruled out.

The diagnostic protocol is the same as in patients without ISM. The risk of skin tests is not greater than in other patients with hymenoptera venom allergy [101]. Specific IgE levels are normally lower than in the general hymenoptera venomallergic population. The results of allergy testing are more frequently negative in up to $15 \%$ of these patients [28], for whom molecular diagnostic or alternative diagnostic tools such as BAT may be beneficial.

Patients with ISM present more serious anaphylaxis and should therefore carry 2 adrenaline auto-injectors.

Immunotherapy is recommended for patients with ISM who have experienced SR and in whom an allergy workup 
Table 4. Spanish Mastocytosis Network (Red Española de Mastocitosis, REMA) Score for Clonal Mast Cell Activation Disorders (MCAD)

\begin{tabular}{lc}
\hline Variable & Score \\
\hline Gender & \\
Male & +1 \\
Female & -1 \\
Clinical Symptoms & \\
Absence of urticaria and angioedema & +1 \\
Urticaria and/or angioedema & -2 \\
Presyncope and/or syncope & +3 \\
Tryptase & \\
$<15 \mathrm{ng} / \mathrm{mL}$ & -1 \\
$>25 \mathrm{ng} / \mathrm{mL}$ & +2 \\
\hline
\end{tabular}

Score $<2$ : low probability of clonal MCAD Score $\geq 2$ : high probability of clonal MCAD

\begin{tabular}{|c|c|}
\hline $\begin{array}{c}\text { Sensitivity: } 0.92 \\
\text { Positive predictive } \\
\text { value: } 0.89\end{array} \quad \begin{array}{c}\text { Specifity: } 0.81 \\
\text { Negative predictive } \\
\text { value: } 0.87\end{array}$ \\
\hline
\end{tabular}

Baseline serum tryptase.

has demonstrated an IgE-mediated mechanism [105-107]. Efficacy ranges from between $14 \%$ and $85 \%$ [108], depending on the study. In any case, it is lower than $80 \%$, less than in other hymenoptera venom allergies. In patients who are not protected after reaching a conventional maintenance dose of $100 \mu \mathrm{g}$, this can be increased to $200 \mu \mathrm{g}$.

There are no studies on long-term efficacy. However, given that the protective effect is lost once VIT is interrupted [109], it is currently accepted that lifelong treatment should be given to patients with ISM. Protocols of extended maintenance with doses every 12-16 weeks are well tolerated by such patients.

Adverse reactions with VIT are somewhat more frequent in patients with ISM than in other patients with hymenoptera venom allergy and affect $23.9 \%$ of patients with ISM [108]. Although adverse reactions are generally more frequent in Vespula VIT, reports from southern Europe show that they are more related to bee venom, as occurs in other hymenoptera venom allergies, mainly in the buildup phase [69]. No data are available on the use of premedication in these patients or on SCT. Extrapolating from data on tolerance and protection with VIT and assuming that such patients are a special group in whom it is important to know the degree of protection, SCT should be performed with the same criteria as in other hymenoptera venom-allergic patients receiving VIT. Nevertheless, extreme caution should be observed in order to adequately treat adverse reactions if they occur.

These patients are at increased risk of anaphylaxis due to stings from other insects and should be advised to always carry adrenaline.

In summary, patients with ISM experience serious anaphylactic reactions, have more SRs with immunotherapy, need to receive lifetime treatment, and, over time, more frequently develop allergies to other hymenoptera. The Spanish Mastocytosis Network (REMA) clonality score makes it possible to identify these patients and correlates with the results from bone marrow punch biopsies. A score of $\geq 2$ is highly specific and sensitive for a high risk for mast cell activation syndrome.

\section{Conclusions}

In this review, we analyze the main diagnostic and therapeutic problems related to hymenoptera venom allergy.

Molecular diagnosis has been a key feature in a large number of publications in recent years and makes it possible to distinguish between cross-reactivity and double sensitization, an issue that is crucial when selecting the most appropriate treatment. However, despite this and other advances, several problems remain to be resolved.

In a register compiled by the Hymenoptera Allergy Committee regarding the prescription of VIT in Spain (internal data), we found that $36.72 \%$ of treatments corresponded to Polistes venom. This figure reflects the importance of this vespid in our setting. However, few Polistes allergens have been identified to date, and Pol d 1, which has been shown to be essential for the characterization of wasp allergy, is not commercially available. Therefore, there is an urgent need to identify and make commercially available new allergens of Polistes, which is a very common species in the Mediterranean area.

It is necessary to conduct more extensive studies to define molecular profiles in patients with poor tolerance to immunotherapy or in whom treatment fails.

The BAT is a promising technique, especially in cases of undetectable IgE, as occurs in patients with systemic mastocytosis, although the technique needs to be standardized and more research is required on its application in clinical practice.

We also need more data on the usefulness of omalizumab as premedication in cases of SR in VIT. Furthermore, the best way to monitor the efficacy of immunotherapy in vitro remains to be defined.

The appearance of new hymenoptera species in our environment, introduced by an extensive network of international transport, may pose diagnostic and therapeutic challenges, as has occurred with some cases of reactions to Vespa velutina in the north of Spain. Given that the real possibility of primary sensitization to this species is low, patients can normally be successfully treated with currently available approaches. However, if the insect manages to become established in Spain, we could be faced with cases of primary sensitization requiring specific VIT.

Finally, allergists must be able to identify patients with ISM associated with anaphylaxis due to hymenoptera stings, as we are the front-line specialists for such cases. Early clinical suspicion and coordination with hematology departments is essential for the correct diagnosis and treatment of this group of patients.

\section{Funding}

The article processing charges were funded by the Foundation of the Spanish Society of Allergy and Clinical Immunology. 


\section{Conflicts of Interest}

The authors declare that they have no conflicts of interest.

\section{References}

1. Fernandez J, Blanca M, Soriano V, Sanchez J, Juarez C. Epidemiological study of the prevalence of allergic reactions to Hymenoptera in a rural population in the Mediterranean area. Clin Exp Allergy. 1999;29:1069-74.

2. Oude Elberink JN, de Monchy JG, Golden DB, Brouwer JL, Guyatt GH, Dubois AE. Development and validation of a healthrelated quality-of-life questionnaire in patients with yellow jacket allergy. J Allergy Clin Immunol. 2002;109:162-70.

3. Armisen M, Guspi R, Alfaya T, Cruz S, Fernandez S, DominguezNoche C, Alonso A, Dalmau G, Marques L, Vega A. CrossSectional Validation of a Quality of Life Questionnaire in Spanish for Patients Allergic to Hymenoptera Venom. J Investig Allergol Clin Immunol. 2015;25:176-82.

4. Golden DB. Anaphylaxis to insect stings. Immunol Allergy Clin North Am. 2015;35:287-302.

5. Bilo BM, Rueff $F$, Mosbech $H$, Bonifazi F, Oude-Elberink JN. Diagnosis of Hymenoptera venom allergy. Allergy. 2005;60:1339-49.

6. Oude Elberink JN, de Monchy JG, van der Heide S, Guyatt GH, Dubois AE. Venom immunotherapy improves health-related quality of life in patients allergic to yellow jacket venom. J Allergy Clin Immunol. 2002;110:174-82.

7. WHO/IUIS Allergen Nomenclature Subcommittee. Allergen nomenclature. 2016. Ref Type: Online Source.

8. Kohler J, Blank S, Muller S, Bantleon F, Frick M, Huss-Marp J, Lidholm J, Spillner E, Jakob T. Component resolution reveals additional major allergens in patients with honeybee venom allergy. J Allergy Clin Immunol. 2014;133:1383-9, 1389.

9. Muller UR, Johansen N, Petersen $A B$, Fromberg-Nielsen J, Haeberli G. Hymenoptera venom allergy: analysis of double positivity to honey bee and Vespula venom by estimation of IgE antibodies to species-specific major allergens Api m1 and Ves v5. Allergy. 2009;64:543-8.

10. Paull BR, Yunginger JW, Gleich GJ. Melittin: an allergen of honeybee venom. J Allergy Clin Immunol. 1977;59:334-8.

11. Ruiz B, Serrano P, Verdu M, Moreno C. Sensitization to Api m 1, Api m 2, and Api m 4: association with safety of bee venom immunotherapy. Ann Allergy Asthma Immunol. 2015;114:350-2.

12. Ruiz B, Serrano P, Verdu M, Moreno C. IgE-Api m4 is useful to identify a particular phenotype of allergy to bee venom. J Investig Allergol Clin Immunol. 2016;26(6).

13. Grunwald T, Bockisch B, Spillner E, Ring J, Bredehorst R, Ollert MW. Molecular cloning and expression in insect cells of honeybee venom allergen acid phosphatase (Api m 3). J Allergy Clin Immunol. 2006;117:848-54.

14. Blank S, Seismann H, Bockisch B, Braren I, Cifuentes L, Mclntyre M, Ruhl D, Ring J, Bredehorst R, Ollert MW, Grunwald T, Spillner E. Identification, recombinant expression, and characterization of the $100 \mathrm{kDa}$ high molecular weight Hymenoptera venom allergens Api m 5 and Ves v 3. J Immunol. 2010;184:5403-13.

15. Van VM, De SL, Rafei-Shamsabadi D, Blank S, Spillner E, Ebo DG, Devreese B, Jakob T, de Graaf DC. IgE recognition of chimeric isoforms of the honeybee (Apis mellifera) venom allergen Api m 10 evaluated by protein array technology. Mol Immunol. 2015;63:449-55.

16. Blank S, Seismann H, Michel Y, McIntyre M, Cifuentes L, Braren I, Grunwald T, Darsow U, Ring J, Bredehorst R, Ollert M, Spillner E. Api $m$ 10, a genuine A. mellifera venom allergen, is clinically relevant but underrepresented in therapeutic extracts. Allergy. 2011;66:1322-9.

17. Monsalve RI, Vega A, Marques L, Miranda A, Fernandez J, Soriano V, Cruz S, Dominguez-Noche C, Sanchez-Morillas L, Armisen-Gil M, Guspi R, Barber D. Component-resolved diagnosis of vespid venom-allergic individuals: phospholipases and antigen $5 \mathrm{~s}$ are necessary to identify Vespula or Polistes sensitization. Allergy. 2012;67:528-36.

18. HemmerW. Cross reactivity in venom allergy: clinical relevance. In: Ollert M, ed. The European Academy Allergy Monographs. Hymenoptera venom allergy. Allergy School Proceedings. 2010:35-9.

19. Ollert M, Blank S. Anaphylaxis to insect venom allergens: role of molecular diagnostics. Curr Allergy Asthma Rep. 2015;15:26.

20. King TP, Spangfort MD. Structure and biology of stinging insect venom allergens. Int Arch Allergy Immunol. 2000;123:99-106.

21. Jin C, Focke $M$, Leonard $R$, Jarisch $R$, Altmann $F$, Hemmer W. Reassessing the role of hyaluronidase in yellow jacket venom allergy. J Allergy Clin Immunol. 2010;125:184-90.

22. Blank S, Seismann H, Mclntyre M, Ollert M, Wolf S, Bantleon $\mathrm{FI}$, Spillner E. Vitellogenins are new high molecular weight components and allergens (Api m 12 and Ves $\vee 6$ ) of Apis mellifera and Vespula vulgaris venom. PLoS One. 2013;8:e62009.

23. Sturm GJ, Hemmer W, Hawranek T, Lang R, Ollert M, Spillner $E$, Blank S, Bokanovic D, Aberer W. Detection of $\lg E$ to recombinant Api $\mathrm{m} 1$ and $\mathrm{rVes} v 5$ is valuable but not sufficient to distinguish bee from wasp venom allergy. J Allergy Clin Immunol. 2011;128:247-8.

24. Korosec P, Silar M, Zidarn M, Kosnik M. Reply: To PMID 22277204. J Allergy Clin Immunol. 2012;130:818-9.

25. Jakob T, Kohler J, Blank S, Magnusson U, Huss-Marp J, Spillner E, Lidholm J. Comparable IgE reactivity to natural and recombinant Api $\mathrm{m} 1$ in cross-reactive carbohydrate determinant-negative patients with bee venom allergy. J Allergy Clin Immunol. 2012;130:276-8.

26. Hofmann SC, Pfender N, Weckesser S, Huss-Marp J, Jakob T. Added value of IgE detection to rApi $\mathrm{m} 1$ and rVes $\vee 5$ in patients with Hymenoptera venom allergy. J Allergy Clin Immunol. 2011;127(1):265-7.

27. Hoffmann HJ, Santos AF, Mayorga C, Nopp A, Eberlein B, Ferrer M, Rouzaire P, Ebo DG, Sabato V, Sanz ML, Pecaric-Petkovic T, Patil SU, Hausmann OV, Shreffler WG, Korosec P, Knol EF. The clinical utility of basophil activation testing in diagnosis and monitoring of allergic disease. Allergy. 2015;70:1393-405.

28. Alvarez-Twose I, Gonzalez de OD, Sanchez-Munoz L, Matito A, Esteban-Lopez MI, Vega A, Mateo MB, Alonso Diaz de Durana MD, de la Hoz B, Del Pozo Gil MD, Caballero T, Rosado A, Sanchez M, I, Teodosio C, Jara-Acevedo M, Mollejo M, Garcia-Montero A, Orfao A, Escribano L. Clinical, biological, and molecular characteristics of clonal mast cell disorders presenting with systemic mast cell activation symptoms. J Allergy Clin Immunol. 2010;125:1269-78. 
29. Golden DB. New directions in diagnostic evaluation of insect allergy. Curr Opin Allergy Clin Immunol. 2014;14:334-9.

30. Vos B, Kohler J, Muller S, Stretz E, Rueff F, Jakob T. Spiking venom with rVes $v 5$ improves sensitivity of IgE detection in patients with allergy to Vespula venom. J Allergy Clin Immunol. 2013;131:1225-7, 1227.

31. Korosec P, Erzen R, Silar M, Bajrovic N, Kopac P, Kosnik M. Basophil responsiveness in patients with insect sting allergies and negative venom-specific immunoglobulin $\mathrm{E}$ and skin prick test results. Clin Exp Allergy. 2009;39:1730-7.

32. Jappe U, Raulf-Heimsoth M, Hoffmann M, Burow G, Hubsch-Muller C, Enk A. In vitro hymenoptera venom allergy diagnosis: improved by screening for cross-reactive carbohydrate determinants and reciprocal inhibition. Allergy. 2006;61:1220-9.

33. Eberlein B, Krischan L, Darsow U, Ollert M, Ring J. Double positivity to bee and wasp venom: improved diagnostic procedure by recombinant allergen-based $\mathrm{IgE}$ testing and basophil activation test including data about crossreactive carbohydrate determinants. J Allergy Clin Immunol. 2012;130:155-61.

34. Carballada FJ, Gonzalez-Quintela A, Nunez-Orjales R, Vizcaino $L$, Boquete M. Double (honeybee and wasp) immunoglobulin E reactivity in patients allergic to Hymenoptera venom: the role of cross-reactive carbohydrates and alcohol consumption. J Investig Allergol Clin Immunol. 2010;20:484-9.

35. Hemmer W, Focke M, Kolarich D, Wilson IB, Altmann F, Wohrl S, Gotz M, Jarisch R. Antibody binding to venom carbohydrates is a frequent cause for double positivity to honeybee and yellow jacket venom in patients with stinging-insect allergy. J Allergy Clin Immunol. 2001;108:1045-52.

36. Hemmer W, Focke M, Kolarich D, Dalik I, Gotz M, Jarisch R. Identification by immunoblot of venom glycoproteins displaying immunoglobulin E-binding N-glycans as crossreactive allergens in honeybee and yellow jacket venom. Clin Exp Allergy. 2004;34:460-9.

37. Kochuyt AM, Van Hoeyveld EM, Stevens EA. Prevalence and clinical relevance of specific immunoglobulin $E$ to pollen caused by sting- induced specific immunoglobulin E to crossreacting carbohydrate determinants in Hymenoptera venoms. Clin Exp Allergy. 2005;35:441-7.

38. Wypych JI, Abeyounis CJ, Reisman RE. Analysis of differing patterns of cross-reactivity of honeybee and yellow jacket venom-specific lgE: use of purified venom fractions. Int Arch Allergy Appl Immunol. 1989;89:60-6.

39. Straumann F, Bucher C, Wuthrich B. Double sensitization to honeybee and wasp venom: immunotherapy with one or with both venoms? Value of FEIA inhibition for the identification of the cross-reacting ige antibodies in double-sensitized patients to honeybee and wasp venom. Int Arch Allergy Immunol. 2000;123:268-74.

40. Caruso B, Bonadonna $P$, Severino MG, Manfredi M, Dama A, Schiappoli M, Rizzotti P, Senna G, Passalacqua G. Evaluation of the IgE cross-reactions among vespid venoms. A possible approach for the choice of immunotherapy. Allergy. 2007;62:561-4.

41. Frick M, Muller S, Bantleon F, Huss-Marp J, Lidholm J, Spillner $\mathrm{E}$, Jakob T. rApi $\mathrm{m} 3$ and $\mathrm{rApi} \mathrm{m} 10$ improve detection of honey bee sensitization in Hymenoptera venom-allergic patients with double sensitization to honey bee and yellow jacket venom. Allergy. 2015;70:1665-8.

42. Muller U, Schmid-Grendelmeier P, Hausmann O, Helbling A. $\lg$ E to recombinant allergens Api $m 1$, Ves $\vee 1$, and Ves $\vee 5$ distinguish double sensitization from crossreaction in venom allergy. Allergy. 2012;67:1069-73.

43. Korosec P, Valenta R, Mittermann I, Celesnik N, Erzen R, Zidarn M, Kosnik M. Low sensitivity of commercially available rApi $\mathrm{m} 1$ for diagnosis of honeybee venom allergy. J Allergy Clin Immunol. 2011;128:671-3.

44. Korosec P, Valenta R, Mittermann I, Celesnik N, Silar M, Zidarn $M$, Kosnik M. High sensitivity of CAP-FEIA rVes $v 5$ and rVes v 1 for diagnosis of Vespula venom allergy. J Allergy Clin Immunol. 2012;129:1406-8.

45. Ebo DG, Van VM, de Graaf DC, Bridts CH, De Clerck LS, Sabato $V$. In vitro diagnosis of Hymenoptera venom allergy and further development of component resolved diagnostics. Expert Rev Clin Immunol. 2014;10:375-84.

46. Moreno C, Barasona MJ, Serrano P, Justicia JL, Ruz JM, Guerra F. Alternating Polistes-Vespula venom immunotherapy: a therapeutic strategy to resolve a diagnostic deficiency. J Investig Allergol Clin Immunol. 2011;21:28-33.

47. Mosbech H, Muller U. Side-effects of insect venom immunotherapy: results from an EAACl multicenter study. European Academy of Allergology and Clinical Immunology. Allergy. 2000;55:1005-10.

48. Antolin-Amerigo D, Moreno AC, Vega A, Alvarez-Mon M. Venom immunotherapy: an updated review. Curr Allergy Asthma Rep. 2014; 14:449.

49. Rueff F, Przybilla B, Bilo MB, Muller U, Scheipl F, Aberer W, Birnbaum J, Bodzenta-Lukaszyk A, Bonifazi F, Bucher C, Campi P, Darsow U, Egger C, Haeberli G, Hawranek T, Korner M, Kucharewicz I, Kuchenhoff H, Lang R, Quercia O, Reider N, Severino M, Sticherling M, Sturm GJ, Wuthrich B. Predictors of severe systemic anaphylactic reactions in patients with Hymenoptera venom allergy: importance of baseline serum tryptase-a study of the European Academy of Allergology and Clinical Immunology Interest Group on Insect Venom Hypersensitivity. J Allergy Clin Immunol. 2009;124:1047-54.

50. Strait RT, Morris SC, Finkelman FD. IgG-blocking antibodies inhibit IgE-mediated anaphylaxis in vivo through both antigen interception and Fc gamma Rllb cross-linking. J Clin Invest. 2006;116:833-41.

51. Rueff F, Przybilla B, Bilo MB, Muller U, Scheipl F, Aberer W, Birnbaum J, Bodzenta-Lukaszyk A, Bonifazi F, Bucher C, Campi P, Darsow U, Egger C, Haeberli G, Hawranek T, Kucharewicz I, Kuchenhoff $H$, Lang R, Quercia O, Reider N, Severino M, Sticherling M, Sturm GJ, Wuthrich B. Predictors of side effects during the buildup phase of venom immunotherapy for Hymenoptera venom allergy: the importance of baseline serum tryptase. J Allergy Clin Immunol. 2010;126:105-11.

52. Brockow K, Kiehn M, Riethmuller C, Vieluf D, Berger J, Ring J. Efficacy of antihistamine pretreatment in the prevention of adverse reactions to Hymenoptera immunotherapy: a prospective, randomized, placebo-controlled trial. J Allergy Clin Immunol. 1997; 100:458-63.

53. Gorska L, Chelminska $M$, Kuziemski K, Skrzypski $M$, Niedoszytko M, Damps-Konstanska I, Szymanowska A, Sieminska A, Wajda B, Drozdowska A, Jutel M, Jassem E. 
Analysis of safety, risk factors and pretreatment methods during rush hymenoptera venom immunotherapy. Int Arch Allergy Immunol. 2008;147:241-5.

54. Schulze J, Rose M, Zielen S. Beekeepers anaphylaxis: successful immunotherapy covered by omalizumab. Allergy. 2007;62:963-4

55. Kontou-Fili K, Filis Cl, Voulgari C, Panayiotidis PG. Omalizumab monotherapy for bee sting and unprovoked "anaphylaxis" in a patient with systemic mastocytosis and undetectable specific IgE. Ann Allergy Asthma Immunol. 2010;104:537-9.

56. Palgan K, Bartuzi Z, Gotz-Zbikowska M. Treatment with a combination of omalizumab and specific immunotherapy for severe anaphylaxis after a wasp sting. Int I Immunopathol Pharmacol. 2014;27:109-12.

57. Tille KS, Parker AL. Imported fire ant rush desensitization using omalizumab and a premedication regimen. Ann Allergy Asthma Immunol. 2014;113:574-6.

58. Bilo MB. Anaphylaxis caused by Hymenoptera stings: from epidemiology to treatment. Allergy. 2011;66 Suppl 95:35-7.

59. Bilo MB, Cinti B, Brianzoni MF, Braschi MC, Bonifazi M, Antonicelli L. Honeybee venom immunotherapy: a comparative study using purified and nonpurified aqueous extracts in patients with normal Basal serum tryptase concentrations. J Allergy (Cairo). 2012;2012:869243.

60. Golden DB. Long-term outcome after venom immunotherapy. Curr Opin Allergy Clin Immunol. 2010;10:337-41.

61. Rueff F, Przybilla B, Bilo MB, Muller U, Scheipl F, Seitz MJ, Aberer W, Bodzenta-Lukaszyk A, Bonifazi F, Campi P, Darsow U, Haeberli G, Hawranek T, Kuchenhoff H, Lang R, Quercia $O$, Reider N, Schmid-Grendelmeier P, Severino M, Sturm GJ, Treudler R, Wuthrich B. Clinical effectiveness of hymenoptera venom immunotherapy: a prospective observational multicenter study of the European academy of allergology and clinical immunology interest group on insect venom hypersensitivity. PLoS One. 2013;8:e63233.

62. Stoevesandt J, Hosp C, Kerstan A, Trautmann A. Hymenoptera venom immunotherapy while maintaining cardiovascular medication: safe and effective. Ann Allergy Asthma Immunol 2015;114:411-6.

63. Rueff $F$, Vos B, Oude EJ, Bender A, Chatelain R, Dugas-Breit $S$, Horny HP, Kuchenhoff $H$, Linhardt A, Mastnik S, Sotlar K, Stretz E, Vollrath R, Przybilla B, Flaig M. Predictors of clinical effectiveness of Hymenoptera venom immunotherapy. Clin Exp Allergy. 2014;44:736-46.

64. Rueff F, Wenderoth A, Przybilla B. Patients still reacting to a sting challenge while receiving conventional Hymenoptera venom immunotherapy are protected by increased venom doses. J Allergy Clin Immunol. 2001;108:1027-32.

65. Lerch E, Muller UR. Long-term protection after stopping venom immunotherapy: results of re-stings in 200 patients. J Allergy Clin Immunol. 1998;101:606-12.

66. Muller U, Helbling A, Berchtold E. Immunotherapy with honeybee venom and yellow jacket venom is different regarding efficacy and safety. J Allergy Clin Immunol. 1992;89:529-35.

67. Muller UR, Ring J. When can immunotherapy for insect sting allergy be stopped? J Allergy Clin Immunol Pract. 2015;3:324-8

68. Zanotti R, Lombardo C, Passalacqua G, Caimmi C, Bonifacio M, De MG, Perbellini O, Rossini M, Schena D, Busa M, Marcotulli
MC, Bilo MB, Franchini M, Marchi G, Simioni L, Bonadonna P. Clonal mast cell disorders in patients with severe Hymenoptera venom allergy and normal serum tryptase levels. J Allergy Clin Immunol. 2015;136:135-9.

69. Bonadonna P, Gonzalez-de-Olano D, Zanotti R, Riccio A, De FL, Lombardo C, Rogkakou A, Escribano L, Alvarez-Twose I, Matito A, Vega A, Passalacqua G. Venom immunotherapy in patients with clonal mast cell disorders: efficacy, safety, and practical considerations. J Allergy Clin Immunol Pract. 2013;1:474-8.

70. Bonadonna P, Bonifacio M, Lombardo C, Zanotti R. Hymenoptera Allergy and Mast Cell Activation Syndromes. Curr Allergy Asthma Rep. 2016;16:5.

71. Cox L, Nelson H, Lockey R, Calabria C, Chacko T, Finegold I, Nelson M, Weber R, Bernstein DI, Blessing-Moore J, Khan DA, Lang DM, Nicklas RA, Oppenheimer J, Portnoy JM, Randolph C, Schuller DE, Spector SL, Tilles S, Wallace D. Allergen immunotherapy: a practice parameter third update. J Allergy Clin Immunol. 2011;127:S1-55.

72. Golden DB, Moffitt J, Nicklas RA, Freeman T, Graft DF, Reisman RE, Tracy JM, Bernstein D, Blessing-Moore J, Cox L, Khan DA, Lang DM, Oppenheimer J, Portnoy JM, Randolph C, Schuller DE, Spector SL, Tilles SA, Wallace D. Stinging insect hypersensitivity: a practice parameter update 2011. J Allergy Clin Immunol. 2011;127:852-4.

73. Ludman SW, Boyle RJ. Stinging insect allergy: current perspectives on venom immunotherapy. J Asthma Allergy. 2015;8:75-86.

74. Bilo BM, Bonifazi F. Hymenoptera venom immunotherapy. Immunotherapy. 2011;3:229-46.

75. Finegold I. Issues in stinging insect allergy immunotherapy: a review. Curr Opin Allergy Clin Immunol. 2008:8:343-7.

76. Bilo MB, Rueff $F$, Mosbech $H$, Bonifazi F, Oude-Elberink JN, Birnbaum J, Bucher C, Muller UR, J.Fernández, Jarish R, Jutel M, Wuthrich B. EAACI Position Paper. Diagnosis of Hymenoptera Venom Allergy. 2005.Ref Type: Online Source.

77. Mobs C, Muller J, Rudzio A, Pickert J, Blank S, Jakob T, Spillner E, Pfutzner W. Decline of Ves v 5-specific blocking capacity in wasp venom-allergic patients after stopping allergen immunotherapy. Allergy. 2015;70:715-9.

78. Erzen R, Kosnik M, Silar M, Korosec P. Basophil response and the induction of a tolerance in venom immunotherapy: a longterm sting challenge study. Allergy. 2012;67:822-30.

79. Cabrera CM, Urra JM, Alfaya T, Roca FL, Feo-Brito F. Expression of Th1, Th2, lymphocyte trafficking and activation markers on CD4+ T-cells of Hymenoptera allergic subjects and after venom immunotherapy. Mol Immunol. 2014;62:178-85.

80. Urra JM, Cabrera CM, Alfaya T, Feo-Brito F. Agreement of skin test with IL-4 production and CD40L expression by T cells upon immunotherapy of subjects with systemic reactions to Hymenoptera stings. Mol Immunol. 2016;70:134-9.

81. Bilo BM, Rueff F. The European Academy Allergy Monographs: Hymenoptera Venom Allergy. 2009.

82. Cortellini $G$, Severino M, Francescato E, Turillazzi S, Spadolini I, Rogkakou A, Passalacqua G. Evaluation and validation of a bee venom sting challenge performed by a micro-syringe. Ann Allergy Asthma Immunol. 2012;109:438-41.

83. Alfaya T, Vega A, Dominguez-Noche C, Ruiz B, Marques L, Sanchez-Morillas L. Longitudinal Validation of the Spanish Version of the Health-Related Quality of Life Questionnaire 
for Hymenoptera Venom Allergy (HRQLHA). J Investig Allergol Clin Immunol. 2015;25:426-30.

84. Fischer J, Teufel M, Feidt A, Giel KE, Zipfel S, Biedermann T. Tolerated wasp sting challenge improves health-related quality of life in patients allergic to wasp venom. J Allergy Clin Immunol. 2013;132:489-90.

85. Koschel DS, Schmies M, Nink Weber C, Höffken G, Balck F. Tolerated Sting Challenge in Patients on Hymenoptera Venom Immunotherapy Improves Health-Related Quality of Life. J Investig Allergol Clin Immunol. 2014;24:226-30.

86. Hoffman DR, El-Choufani SE, Smith MM, de GH. Occupational allergy to bumblebees: allergens of Bombus terrestris. J Allergy Clin Immunol. 2001;108:855-60.

87. The Allergome Database. 2016. Ref Type: Online Source.

88. Van VM, Debyser G, Smagghe G, Devreese B, de Graaf DC. Unraveling the venom proteome of the bumblebee (Bombus terrestris) by integrating a combinatorial peptide ligand library approach with FT-ICR MS. Toxicon. 2015;102:81-8.

89. Müeller U. Insect sting allergy: clinical picture, diagnostic and treatment. Sttutgart-New York: Gustav Fisher Verlag, 1990.

90. Stern A, Wuthrich B, Mullner G. Successful treatment of occupational allergy to bumblebee venom after failure with honeybee venom extract. Allergy. 2000;55:88-91.

91. Fernández S, Baltasar M. Inmunoterapia con himenópteros: doble sensibilización. J Investig Allergol Clin Immunol. 2008; 18(Suppl.3):73-5.

92. Cruz $S$, Vega A, Fernandez S, Marques L, Baltasar M, Alonso A, Jorro G, Moreno A, Sanchez-Morillas L, Miranda A, Soriano V, Fernandez J, Guspi R. Report from the Hymenoptera Committee of the Spanish Society of Allergology and Clinical Immunology: immunotherapy with bumblebee venom. J Investig Allergol Clin Immunol. 2012;22:377-8.

93. Bonifazi F, Jutel M, Bilo BM, Birnbaum J, Muller U. Prevention and treatment of hymenoptera venom allergy: guidelines for clinical practice. Allergy. 2005;60:1459-70.

94. Antonicelli L, Bilo MB, Napoli G, Farabollini B, Bonifazi F. European hornet (Vespa crabro) sting: a new risk factor for life-threatening reaction in hymenoptera allergic patients? Eur Ann Allergy Clin Immunol. 2003; 35:199-203.

95. Chugo S, Lizaso MT, Alvarez MJ, Arroabaren E, Lizarza S, Tabar Al. Vespa velutina nigritorax: A New Causative Agent in Anaphylaxis. J Investig Allergol Clin Immunol. 2015;25:231-2.

96. Goldberg A, Shefler I, Panasoff J, Paitan Y, Confino-Cohen R. Immunotherapy with commercial venoms is efficacious for anaphylactic reactions to Vespa orientalis stings. Int Arch Allergy Immunol. 2013;161:174-80.

97. Sastre J. Molecular diagnosis in allergy. Clin Exp Allergy. 2010; 40:1442-60.

98. Severino MG, Caruso B, Bonadonna P, Labardi D, Macchia D, Campi P, Passalacqua G. Cross reactivity between European hornet and yellow jacket venoms. Eur Ann Allergy Clin Immunol. 2010;42:141-5.

99. Brockow K, Jofer C, Behrendt H, Ring J. Anaphylaxis in patients with mastocytosis: a study on history, clinical features and risk factors in 120 patients. Allergy. 2008;63:226-32.

100. Bonadonna P, Zanotti R, Pagani M, Caruso B, Perbellini O, Colarossi S, Olivieri E, Dama A, Schiappoli M, Senna G, Antico A, Passalacqua G. How much specific is the association between hymenoptera venom allergy and mastocytosis? Allergy. 2009;64:1379-82.
101. Gonzalez de OD, de la Hoz CB, Nunez LR, Sanchez ML, Cuevas AM, Dieguez MC, Alvarez Twose I, Castells MC, Escribano ML. Prevalence of allergy and anaphylactic symptoms in 210 adult and pediatric patients with mastocytosis in Spain: a study of the Spanish network on mastocytosis (REMA). Clin Exp Allergy. 2007;37:1547-55.

102. Bonadonna P, Perbellini O, Passalacqua G, Caruso B, Colarossi S, Dal FD, Castellani L, Bonetto C, Frattini F, Dama A, Martinelli G, Chilosi M, Senna G, Pizzolo G, Zanotti R. Clonal mast cell disorders in patients with systemic reactions to Hymenoptera stings and increased serum tryptase levels. J Allergy Clin Immunol. 2009;123:680-6.

103. Alvarez-Twose I, Zanotti R, Gonzalez-de-Olano D, Bonadonna P, Vega A, Matito A, Sanchez-Munoz L, Morgado JM, Perbellini O, Garcia-Montero A, De MG, Teodosio C, Rossini M, JaraAcevedo M, Schena D, Mayado A, Zamo A, Mollejo M, SanchezLopez P, Cabanes N, Orfao A, Escribano L. Nonaggressive systemic mastocytosis (SM) without skin lesions associated with insect-induced anaphylaxis shows unique features versus other indolent SM. J Allergy Clin Immunol. 2014;133:520-8.

104. Alvarez-Twose I, Gonzalez-de-Olano D, Sanchez-Munoz L, Matito A, Jara-Acevedo M, Teodosio C, Garcia-Montero A, Morgado JM, Orfao A, Escribano L. Validation of the REMA score for predicting mast cell clonality and systemic mastocytosis in patients with systemic mast cell activation symptoms. Int Arch Allergy Immunol. 2012;157:275-80.

105. Gonzalez-de-Olano D, Alvarez-Twose I, Vega A, Orfao A, Escribano L. Venom immunotherapy in patients with mastocytosis and hymenoptera venom anaphylaxis. Immunotherapy. 2011;3:637-51.

106. Fricker M, Helbling A, Schwartz L, Muller U. Hymenoptera sting anaphylaxis and urticaria pigmentosa: clinical findings and results of venom immunotherapy in ten patients. J Allergy Clin Immunol. 1997;100:11-5.

107. Haeberli G, Bronnimann M, Hunziker T, Muller U. Elevated basal serum tryptase and hymenoptera venom allergy: relation to severity of sting reactions and to safety and efficacy of venom immunotherapy. Clin Exp Allergy 2003;33:1216-20.

108. Niedoszytko M, Bonadonna P, Oude Elberink JN, Golden DB. Epidemiology, diagnosis, and treatment of Hymenoptera venom allergy in mastocytosis patients. Immunol Allergy Clin North Am. 2014;34:365-81.

109. Oude Elberink JN, de Monchy JG, Kors JW, van Doormaal JJ, Dubois AE. Fatal anaphylaxis after a yellow jacket sting, despite venom immunotherapy, in two patients with mastocytosis. J Allergy Clin Immunol. 1997;99:153-4.

\section{Manuscript received November 16, 2016; accepted for publication December 5, 2016.}

\section{Teresa Alfaya}

\author{
Servicio de Alergia \\ Hospital General Universitario de Ciudad Real \\ C/Obispo Rafael Torija s/n \\ 13005 Ciudad Real \\ E-mail: talfaya@sescam.jccm.es
}

